\title{
Identification of genes associated with osteoarthritis by microarray analysis
}

\author{
JIANWEI SUN, BINGSHAN YAN, WANGPING YIN and XINCHAO ZHANG
}

\author{
Department of Orthopedics, Jinshan Hospital of Fudan University, Shanghai 201508, P.R. China
}

Received October 21, 2014; Accepted June 15, 2015

DOI: $10.3892 / \mathrm{mmr} .2015 .4048$

\begin{abstract}
The aim of the present study was to investigate the mechanisms of osteoarthritis (OA). Raw microarray data (GSE51588) were downloaded from Gene Expression Omnibus, including samples from OA $(n=20)$ and non-OA $(n=5)$ knee lateral and medial tibial plateaus. Differentially expressed genes (DEGs) were identified using Student's t-test. Functional and pathway enrichment analyses were performed for the upregulated and downregulated DEGs. A protein-protein interaction network (PPI) was constructed according to the Search Tool for the Retrieval of Interacting Genes/Proteins database, and module analysis of the PPI network was performed using CFinder. The protein domain enrichment analysis for genes in modules was performed using the INTERPRO database. A total of 869 upregulated and 508 downregulated DEGs were identified. The enriched pathways of downregulated and upregulated DEGs were predominantly associated with the cell cycle (BUB1, BUB1B, CCNA2, CCNB1 and CCNE1), and extracellular matrix (ECM)-receptor interaction (CD36, COL11A2, COL1A1, COL2A1 and COL3A1). Functional enrichment analysis of the DEGs demonstrated that FGF19, $K I F 11$ and $K I F 2 C$ were involved in the response to stress and that $A C A N, A D A M T S 10$ and $B G N$ were associated with proteinaceous ECM. The top protein domain was IPR001752: Kinesin motor region involving three genes (KIF2C, KIF11 and KIF20A). The identified DEGs, including KIF2C, KIF11 and $K I F 20 A$, may be significant in the pathogenesis of OA.
\end{abstract}

\section{Introduction}

Osteoarthritis (OA) is the most prevalent chronic joint disease and results in a large economic burden due to the associated costs of medical care and lost earnings (1). OA is characterized

Correspondence to: Dr Wangping Yin or Dr Xinchao Zhang, Department of Orthopedics, Jinshan Hospital of Fudan University, 1508 Longhang Road, Shanghai 201508, P.R. China

E-mail:wangpingyinn@163.com

E-mail: zhangxc410@aliyun.com

Key words: osteoarthritis, enrichment analysis, module analysis, protein domain enrichment analysis by the disappearance of the cartilage, combined with sub-chondral bone sclerosis, formation of osteophytes and a mild inflammation of the synovial membrane (2).

The pathology of OA is complex and a large number of studies have focused on the pathogenesis of OA to identify a therapeutic method for patients with OA. The sub-chondral bone is involved in the pathophysiology of OA through biochemical and mechanical pathways (3). OA microarray analysis has predominantly focused on the articular cartilage, meniscus or synovium; however, only few studies on sub-chondral bone are available $(4,5)$. Therefore, the present study assessed the sub-chondral bone in order to enhance the understanding of the pathology of OA. Zhen et al (6) reported that the pathological changes of OA may be caused by high concentrations of active transforming growth factor- $\beta 1$ in the subchondral bone, and that inhibition of this process may represent a potential therapeutic method. According to a study by Valverde-Franco et al (7), bone-specific overexpression of EphB4 on subchondral bone and cartilage has a protective effect on OA. With the number of studies increasing, further genes associated with $\mathrm{OA}$ are being identified.

Microarray analysis has been used to analyze the gene expression of thousands of transcripts in OA samples. Several novel candidate genes, including bone formation-associated genes (CLEC3B, CDH11, GPNMB, CLEC3A, CHST11, $M S X 1, M S X 2)$ and genes encoding collagens (COL13A1, COL14A1, COL15A1, COL8A2), were identified by microarray analysis (8). Chou et al (4) only performed functional and pathway enrichment analyses of the identified differentially expressed genes (DEGs). In the present study, the microarray data (GSE51588) (4) were downloaded from Gene Expression Omnibus using additional bioinformatics analysis to gain further insight into the molecular mechanisms of OA. Gene Ontology (GO) functional terms and Kyoto Encyclopedia of Genes and Genomes (KEGG) pathway enrichment analysis were performed for the DEGs. In addition, a protein-protein interaction (PPI) network was constructed and protein domain enrichment of the genes in the modules of the PPI network were performed to screen the significant genes, which were involved in the pathogenesis of OA.

\section{Materials and methods}

Microarray data and data pre-processing. The microarray data (GSE51588) deposited by Chou et al (4) were downloaded 
from the Gene Expression Omnibus database (http://www. ncbi.nlm.nih.gov/geo/). The platform used was the GPL13497 Agilent-026652 Whole Human Genome Microarray 4x44 K v2 (Agilent Technologies, Palo Alto, CA, USA). A total of 50 samples were available, including 20 OA knee lateral tibial plateau samples, 20 OA knee medial plateau samples, 5 non-OA knee lateral tibial plateau samples and 5 non-OA knee medial plateau samples.

For data pre-processing, the expression profile chip was pre-processed using the Affy package in Bioconductor (http://www.bioconductor.org/packages/release/bioc/html/affy.html) (9) and Affymetrix annotation files from Brain Array Lab (Affymetrix, Santa Clara, CA, USA; http://www.affymetrix.com/analysis/). The background correction, quartile data normalization and probe summarization were performed using the robust multiarray average algorithm (http://www.bioconductor.org) (10) to obtain a gene expression matrix.

Identification of DEGs. The expression values for the normalized data were calculated using the Limma package in R (Affymetrix; http://www.affymetrix.com/analysis/) (11). DEGs were identified by Student's t-test. The raw P-value was adjusted into false discovery rate (FDR) using the Benjamini \& Hochberg method (12). An FDR $<0.01$ and $\mid \log 2$ fold changel $>1$ were selected as cut-off criteria.

Enrichment analysis for DEGs. Functional enrichment of the DEGs in the biological process, molecular function and cellular component categories was performed using the GO database (http://geneontology.org/) (13). Based on the KEGG database (http://www.genome.jp/kegg/pathway.html) (14), pathway enrichment of the DEGs was performed. $\mathrm{P}<0.01$ was selected as a cut-off criterion.

Construction of the PPI network and module analysis. The identified DEGs were mapped to the Search Tool for the Retrieval of Interacting Genes version 9.1 database (http://www.string-db.org/) (15) to search for the interaction associations between the proteins, and a confidence score $>0.4$ was selected as cut-off criterion. Cytoscape software (http://www.cytoscape.org/) (16) was subsequently used to visualize the PPI network.

Module analysis of the PPI network was performed by CFinder (http://www.cfinder.org/) (17). The search algorithm using the Clique Percolation Method was used to identify the k-clique percolation clusters of the network (18). Larger k-clique values represented higher stringency during the identification of dense groups and provided smaller groups with a higher density of links inside them. A k-cliques value of 5 was selected as the cut-off criterion. The protein domain enrichment analysis of genes in the module was analyzed using the INTERPRO database (http://www. ebi.ac.uk/interpro/) $(17,19)$ and $\mathrm{P}<0.05$ was selected as the cut-off criterion.

\section{Results}

DEG analysis. For microarray analysis, 1,377 DEGs were identified between the OA group and the non-OA group. Of these genes, 869 DEGs were upregulated and 508 DEGs were downregulated.

Functional and pathway enrichment analysis of the DEGs. The functional enrichment analysis of the downregulated DEGs revealed that the top five enriched pathways were associated with systemic lupus erythematosus $\left(\mathrm{P}=1.17 \times 10^{-13}\right)$, the cell cycle $\left(\mathrm{P}=1.47 \times 10^{-6}\right)$, complement and coagulation cascades $(\mathrm{P}=0.000685)$, nitrogen metabolism $(\mathrm{P}=0.001086)$ and amoebiasis ( $\mathrm{P}=0.001278$ ) (Table I). $B U B 1, B U B 1 B, C C N A 2$, $C C N B 1$ and $C C N E 1$ were the genes involved in the cell cycle. The top five enriched GO terms included response to stress $\left(\mathrm{P}=4.91 \times 10^{-14}\right)$, involving CCNA2, CCNB1, FGF19, KIF11 and $K I F 2 C$, immune system-associated processes $\left(\mathrm{P}=1.87 \times 10^{-12}\right)$ involving $F G F 19, K I F 11$ and $K I F 2 C$, response to wounding $\left(\mathrm{P}=2.04 \times 10^{-12}\right)$, including $C C N B 1, K I F 11$ and $K I F 2 C$, defense response $\left(\mathrm{P}=2.37 \times 10^{-12}\right)$, involving $F G F 19$, and mitotic cell cycle $\left(\mathrm{P}=2.60 \times 10^{-12}\right)$ associated with $B U B 1, B U B 1 B, C C N A 2$, CCNB1, CCNE1, KIF20, KIF11 and KIF2C (Table II).

The functional enrichment analysis of the upregulated DEGs demonstrated that the top five enriched pathways were associated with extracellular matrix (ECM)-receptor interaction $\left(\mathrm{P}=3.01 \times 10^{-5}\right)$, axon guidance $(\mathrm{P}=0.000472)$, amoebiasis $(\mathrm{P}=0.003458)$, focal adhesion $(\mathrm{P}=0.003709)$ and cancer-associated pathways $(\mathrm{P}=0.00407)$ (Table III). Certain genes, including CD36, COL11A2, COL1A1, COL2A1 and COL3A1, were involved in the ECM-receptor interaction pathway. In addition, the $\mathrm{GO}$ functional enrichment analysis demonstrated that $A C A N, A D A M T S 10$ and $B G N$ were involved in proteinaceous $\operatorname{ECM}\left(\mathrm{P}=1.11 \times 10^{-15}\right)$ and $\operatorname{ECM}\left(\mathrm{P}=2.22 \times 10^{-15}\right)$ terms, $A 2 M, A B C C 6$ and $A C A N$ were involved in single-multicellular organism process $\left(\mathrm{P}=4.66 \times 10^{-15}\right)$, and multicellular organism process $\left(\mathrm{P}=8.27 \times 10^{-14}\right)$ terms, while ADAMTS10, COL10A1 and $C O L 11 A 2$ were involved in the ECM part $\left(\mathrm{P}=5.17 \times 10^{-14}\right)$ (Table IV).

Analysis of PPI network and modules. Two modules, including module 2 (Fig. 1) and module 5 (Fig. 2) were extracted from the constructed PPI network. Protein domain enrichment analysis revealed that the genes in module 5 were not significantly enriched in any protein domain. A total of 10 protein domains were enriched for the genes in module 2 (Table V), including IPR001752: Kinesin, motor region $(\mathrm{P}=0.001518)$ involving three genes $(K I F 2 C, K I F 11$ and KIF20A), IPR013212: Mad3/BUB1 homology region 1 $(\mathrm{P}=0.002759)$ associated with two genes $(B U B 1$ and $B U B 1 B)$ and IPR014400: Cyclin A/B/D/E (P=0.016448) involving two genes $(C C N B 1$ and $C C N A 2)$.

\section{Discussion}

In the present study, the gene expression profiles of the OA group and the non-OA group were analyzed and 1,377 DEGs were identified, including 869 upregulated DEGs and 508 downregulated DEGs. The functional enrichment for the downregulated DEGs revealed that the FGF19 gene was involved in the response to stress as well as the defense response. According to Tew et al (20), apoptosis and proliferation are involved in the progression of cartilage wounding during the development of OA. Costouros and Kim (21) 
Table I. Top five enriched KEGG pathways for downregulated differentially expressed genes.

\begin{tabular}{lccl}
\hline KEGG pathway & Gene count & P-value & Genes \\
\hline Systemic lupus erythematosus & 27 & $1.17 \times 10^{-13}$ & $C 9, C T S G, E L A N E, H 2 A F X, H I S T 1 H 2 A C$ \\
Cell cycle & 17 & $1.47 \times 10^{-6}$ & $B U B 1, B U B 1 B, C C N A 2, C C N B 1, C C N E 1$ \\
Complement and coagulation cascades & 9 & 0.000685 & $C 5 A R 1, C 9, C R 1, F 12, F 3$ \\
Nitrogen metabolism & 5 & 0.001086 & $C A 1, C A 2, C A 8, G L U L, H A L$ \\
Amoebiasis & 11 & 0.001278 & ARG1, ARG2,C9,COL4A6,CTSG \\
\hline
\end{tabular}

KEGG, Kyoto Encyclopedia of Genes and Genomes.

Table II. Top five enriched GO terms for the downregulated differentially expressed genes.

\begin{tabular}{|c|c|c|c|c|}
\hline GO ID & Term & $\begin{array}{l}\text { Gene } \\
\text { count }\end{array}$ & P-value & Genes \\
\hline 0006950 & Response to stress & 155 & $4.91 \times 10^{-14}$ & KIF11, KIF2C, FGF19, ADORA3, AEN \\
\hline 0002376 & Immune system process & 111 & $1.87 \times 10^{-12}$ & KIF11, KIF2C, FGF19, АНCY, АРОВЕС $3 B$ \\
\hline 0009611 & Response to wounding & 75 & $2.04 \times 10^{-12}$ & KIF11, KIF2C, ADM, ADORA3, AHCY \\
\hline 0006952 & Defense response & 85 & $2.37 \times 10^{-12}$ & FGF19, AHCY, ALOX5, ALOX5AP, APOBEC $3 B$ \\
\hline 0000278 & Mitotic cell cycle & 61 & $2.60 \times 10^{-12}$ & KIF11, KIF2C, KIF20A, AURKA, AURKB \\
\hline
\end{tabular}

GO, gene ontology.

Table III. Top five enriched KEGG pathways for the upregulated differentially expressed genes.

\begin{tabular}{|c|c|c|c|}
\hline KEGG pathway & Gene count & P-value & Genes \\
\hline ECM-receptor interaction & 12 & $3.01 \times 10^{-5}$ & CD36, COL11A2, COL1A1, COL2A1, COL3A1 \\
\hline Axon guidance & 13 & 0.000472 & EPHB3, LRRC4C,NTN1, NTN4, PLXNA3 \\
\hline Amoebiasis & 10 & 0.003458 & COL11A2, COL1A1, COL2A1, COL3A1, COL4A5 \\
\hline Focal adhesion & 15 & 0.003709 & AKT2,COL11A2,COL1A1, COL2A1,COL3A1 \\
\hline Pathways in cancer & 21 & 0.00407 & $A K T 2, A R N T 2, C O L 4 A 5, C S F 1 R, D A P K 2$ \\
\hline
\end{tabular}

KEGG, Kyoto Encyclopedia of Genes and Genomes; ECM, extracellular matrix.

Table IV. Top five enriched GO terms for the upregulated differentially expressed genes.

\begin{tabular}{llcll}
\hline GO ID & \multicolumn{1}{c}{ Term } & Gene count & P-value & Genes \\
\hline 0005578 & Proteinaceous extracellular matrix & 51 & $1.11 \times 10^{-15}$ & ACAN, ADAMTS10, BGN, C1QTNF8, C1QT- \\
NF9 & & & \\
0031012 & Extracellular matrix & 55 & $2.22 \times 10^{-15}$ & $A C A N, A D A M T S 10, B G N, C 1 Q T N F 8, C 1 Q T N F 9$ \\
0044707 & Single-multicellular organism process & 305 & $4.66 \times 10^{-15}$ & A2M, ABCC6,ACAN,ADAM20,ADAP2 \\
0044420 & Extracellular matrix part & 33 & $5.17 \times 10^{-14}$ & ADAMTS10,C1QTNF8,C1QTNF9,COL10A1/2 \\
0032501 & Multicellular organismal process & 309 & $8.27 \times 10^{-14}$ & A2M, ABCC6,ACAN,ADAM20,ADAP2 \\
\hline
\end{tabular}

GO, gene ontology.

reported that the use of programmed cell death inhibitors can improve the results of cartilage repair surgeries. fibroblast growth factor (FGF)2 is a mitogen that induces chondrocyte proliferation in wounded cartilage (22). FGF18 induces the increase of cartilage thickness of the tibial plateau and also increases remodeling of the subchondral bone by repairing 


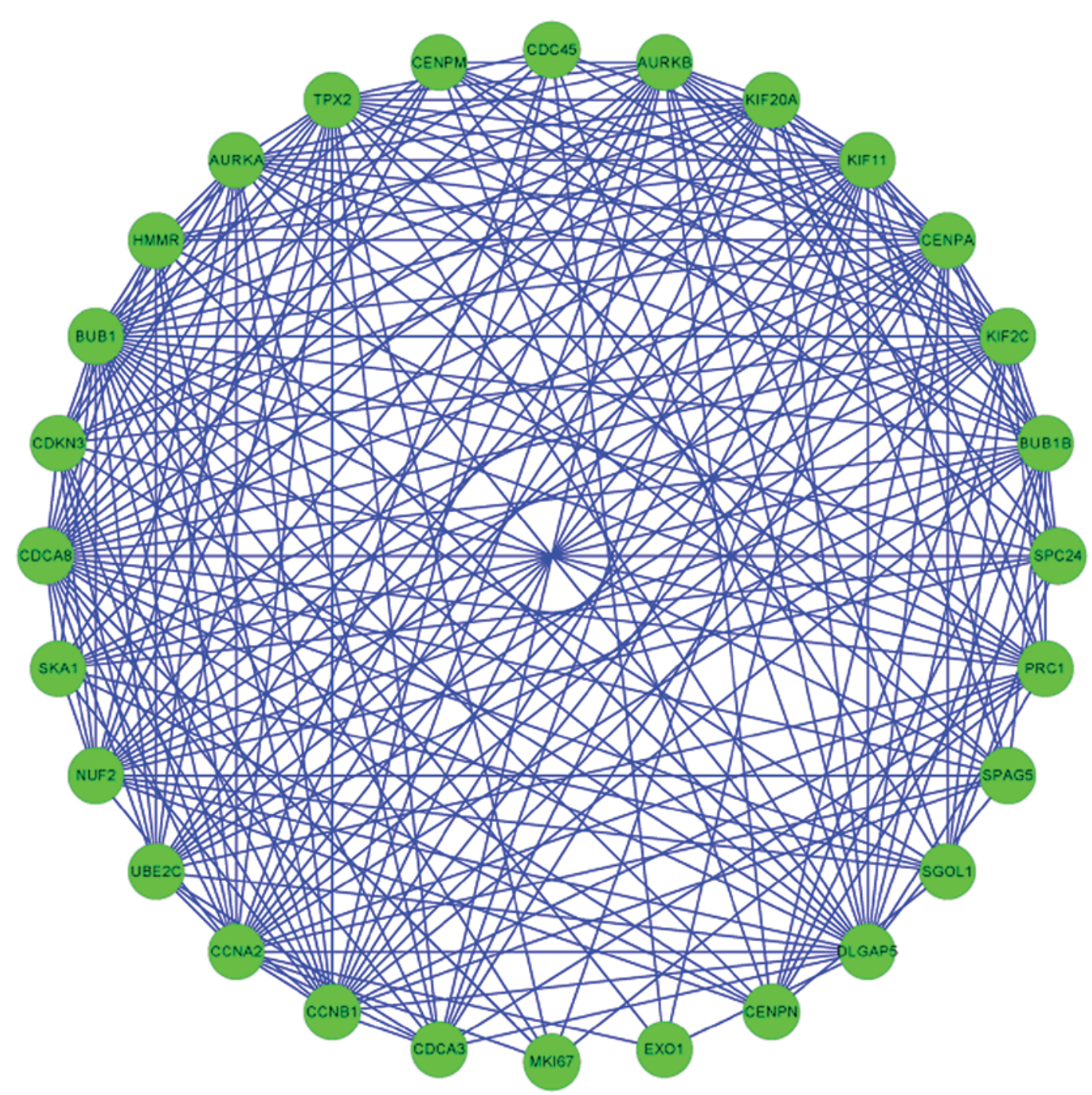

Figure 1. Module 2 of protein-protein interaction network of the downregulated differentially expressed genes.

damaged cartilage in OA rats (23). FGF2 and FGF18 are involved in cartilage wounding, which may be considered as one of the responses to stress. Therefore, the present study hypothesized that FGF19 may be involved in the repair of damaged cartilage in OA.

Functional enrichment analysis of the downregulated DEGs further revealed that KIFII and KIF2C were involved in the response to stress, immune system process, response to wounding and mitotic cell cycle. In addition, the protein domain enrichment analysis of the genes in module 2 showed that KIF2C, KIF11 and KIF20A, were significantly enriched in the protein domain of IPR001752: Kinesin, motor region. KIF2C, KIFII and KIF2OA belong to the Kinesin family of proteins. Kinesins are important in cell division and at least 12 kinesins are involved in mitosis and cytokinesis (24). KIF 22 is a member of the kinesin-like protein family and KIF22 mRNA was detected in human bone, cartilage, joint capsules, ligaments, skin and primary cultured chondrocytes (25). Mutations in KIF22 may be the cause of spondyloepimetaphyseal dysplasia with joint laxity, which is an autosomal dominant skeletal disorder (26). Phosphocitrate is known to downregulate multiple genes responsible for cell proliferation. KIF 23 and $K I F C l$ were shown to be differentially expressed between

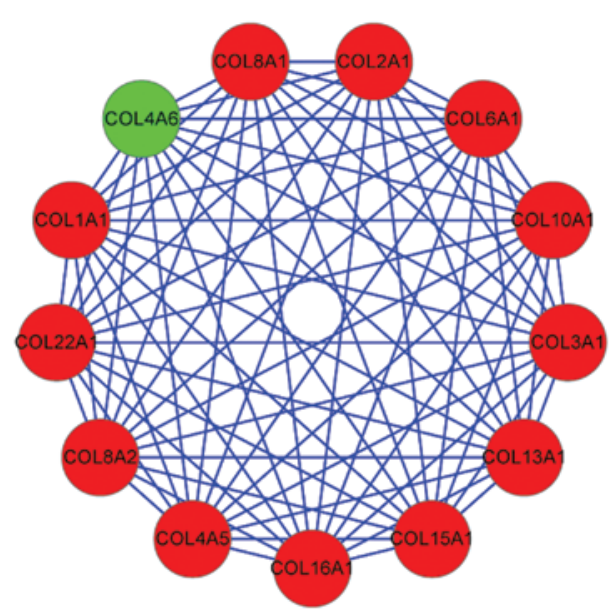

Figure 2. Module 5 of protein-protein interaction network. The red nodes illustrate upregulated differentially expressed genes and the green nodes illustrate downregulated differentially expressed genes.

phosphocitrate-treated and -untreated telomerase-transduced OA 13A fibroblast-like synoviocytes (27). KIF2C was also identified as a DEG in phosphocitrate-treated vs. untreated 
Table V. Enriched protein domains for genes in module 2 of the protein-protein interaction network (category, Interpro).

\begin{tabular}{|c|c|c|c|c|}
\hline Term & Name/function & Count & P-value & Genes \\
\hline IPR001752 & Kinesin, motor region & 3 & 0.001518 & $K I F 2 C, K I F 11, K I F 20 A$ \\
\hline IPR019821 & Kinesin, motor region, conserved site & 3 & 0.001518 & $K I F 2 C, K I F 11, K I F 20 A$ \\
\hline IPR013212 & Mad3/BUB1 homology region 1 & 2 & 0.002759 & $B U B 1, B U B 1 B$ \\
\hline IPR015661 & $\begin{array}{l}\text { Mitotic checkpoint, serine/threonine } \\
\text { protein kinase, BUB1 }\end{array}$ & 2 & 0.002759 & $B U B 1, B U B 1 B$ \\
\hline IPR008271 & $\begin{array}{l}\text { Serine/threonine protein kinase, } \\
\text { active site }\end{array}$ & 4 & 0.012294 & $B U B 1, B U B 1 B, A U R K A, A U R K B$ \\
\hline IPR014400 & Cyclin A/B/D/E & 2 & 0.016448 & $C C N B 1, C C N A 2$ \\
\hline IPR004367 & Cyclin, C-terminus & 2 & 0.019164 & $C C N B 1, C C N A 2$ \\
\hline IPR017441 & Protein kinase, ATP binding site & 4 & 0.023893 & $B U B 1, B U B 1 B, A U R K A, A U R K B$ \\
\hline IPR006671 & Cyclin, N-terminus & 2 & 0.044611 & $C C N B 1, C C N A 2$ \\
\hline IPR013763 & Cyclin-associated & 2 & 0.049887 & $C C N B 1, C C N A 2$ \\
\hline
\end{tabular}

IPR, interpro; ATP, adenosine triphosphate.

OA meniscal cells (28). These results suggested that $K I F 2 C$, KIF11 and KIF20A may be involved in the mitotic cell cycle and contribute to the development of OA.

In addition, functional enrichment analysis of the downregulated DEGs revealed that $B U B 1, B U B 1 B, C C N B 1$ and $C C N A 2$ were involved in the mitotic cell cycle process. Furthermore, the protein domain enrichment analysis of the genes in module 2 , including $B U B 1$ and $B U B 1 B$, revealed that these genes were significantly enriched in the protein domain of IPR013212: Mad3/BUB1 homology region (1), and the genes in module 2, including CCNB1 and CCNA2, were significantly enriched in the protein domain of IPR014400: Cyclin A/B/D/E. Several previous studies have reported that $B U B 1, B U B 1 B, C C N B 1$ and $C C N A 2$ had roles in the regulation of the cell cycle (29-32). According to Tew et al (20), apoptosis and proliferation were involved in processes of cartilage wounding. Based on these results, the present study hypothesized that $B U B 1, B U B 1 B, C C N B 1$ and $C C N A 2$ affected the development of OA through the cell cycle.

In addition, upregulated DEGs, including CD36, COL11A2, COL1A1, COL2A1 and COL3A1, were revealed to be involved in the ECM and ECM-receptor interaction pathway. The structural matrix macromolecules in the ECM and growth factors regulate the chondrocyte function via specific membrane receptors (33). TSP-1 is involved in the cell-matrix interactions of various tissues in cartilage. The number of $C D 36$-positive chondrocytes, which is considered to be the receptor of TSP-1, is significantly increased in severely osteoarthritic cartilage (34). The expression of $C D 36$ patterning receptor has attenuating effects on the inflammatory, pro-catabolic responses to S100A11 and tumor necrosis factor $\alpha$ in chondrocytes (35). The $\alpha 1(\mathrm{X})$ collagen gene COL10A1 is a hypertrophic chondrocytes-specific molecular marker and a transcriptional target of RUNX2 during chondrogenesis $(36,37)$. RUNX2 also regulates the expression of COL1OA1 in hypertrophic chondrocytes and matrix metallopeptidase 13 in terminal hypertrophic chondrocytes (38). The collagen genes (COL11A2, COL1A1 and COL2A1) are involved in the progression of $\mathrm{OA}$ through the mediation of sensitivity to OA (39). The upregulated expression of COL1A1 and COL2A1 reflects the metabolic activation of OA chondrocytes, and the expression of COL11A2 is also upregulated with the progression of OA (40). The results suggested that the DEGs associated with the ECM are important in the regulation of chondrocyte function.

In conclusion, in the present study, gene expression analysis was performed and 1,377 DEGs were identified, among which 869 DEGs were upregulated and 508 DEGs were downregulated. The identified DEGs may be involved in the pathogenesis of OA, particularly FGF19, BUB1, KIF2C, $C D 36$ and $C O L 11 A 2$, which are involved in the cellular stress response, cell cycle and the ECM. However, the results of the present study require confirmation by further studies.

\section{References}

1. Fransen M, Bridgett L, March L, Hoy D, Penserga E and Brooks P: The epidemiology of osteoarthritis in Asia. Int J Rheum Dis 14: 113-121, 2011.

2. Mahjoub M, Berenbaum F and Houard X: Why subchondral bone in osteoarthritis? The importance of the cartilage bone interface in osteoarthritis. Osteoporos Int 23 (Suppl 8): S841-S846, 2012.

3. Henrotin Y, Pesesse L and Sanchez C: Subchondral bone and osteoarthritis: Biological and cellular aspects. Osteoporos Int 23: S847-S851, 2012.

4. Chou CH, Wu CC, Song IW, Chuang HP, Lu LS, Chang JH, Kuo SY, Lee CH, Wu JY, Chen YT, et al: Genome-wide expression profiles of subchondral bone in osteoarthritis. Arthritis Res Ther 15: R190, 2013.

5. Chou CH, Wu CC, Song IW, Chuang HP, Lu LS, Chang JH, Kuo SY, Lee CH, Wu JY and Chen YT: Genome-wide expression profiles of subchondral bone in osteoarthritis. Arthritis Res Ther 15: R190, 2013.

6. Zhen G, Wen C, Jia X, Li Y, Crane JL, Mears SC, Askin FB, Frassica FJ, Chang W, Yao J, et al: Inhibition of TGF- $\beta$ signaling in mesenchymal stem cells of subchondral bone attenuates osteoarthritis. Nat Med 19: 704-712, 2013.

7. Valverde-Franco G, Pelletier JP, Fahmi H, Hum D, Matsuo K, Lussier B, Kapoor M and Martel-Pelletier J: In vivo bone-specific EphB4 overexpression in mice protects both subchondral bone and cartilage during osteoarthritis. Arthritis Rheum 64: 3614-3625, 2012.

8. Karlsson C, Dehne T, Lindahl A, Brittberg M, Pruss A, Sittinger $\mathrm{M}$ and Ringe J: Genome-wide expression profiling reveals new candidate genes associated with osteoarthritis. Osteoarthritis Cartilage 18: 581-592, 2010. 
9. Gautier L, Cope L, Bolstad BM and Irizarry RA: Affy-analysis of Affymetrix GeneChip data at the probe level. Bioinformatics 20: 307-315, 2004

10. Irizarry RA, Hobbs B, Collin F, Beazer-Barclay YD, Antonellis KJ, Scherf U and Speed TP: Exploration, normalization and summaries of high density oligonucleotide array probe level data. Biostatistics 4: 249-264, 2003.

11. Smyth G and Limma: Linear models for microarray data Bioinformatics and Computational Biology Solutions using $\mathrm{R}$ and Bioconductor 2005. Springer, New York, pp 397-420, 2005.

12. Benjamini Y and Hochberg Y: Controlling the false discovery rate: A practical and powerful approach to multiple testing. J R Stat Soc 57: 289-300, 1995.

13. Ashburner M, Ball CA, Blake JA, Botstein D, Butler H, Cherry JM, Davis AP, Dolinski K, Dwight SS, Eppig JT, et al: Gene Ontology: Tool for the unification of biology. The gene ontology consortium. Nat Genet 25: 25-29, 2000.

14. Kanehisa M and Goto S: KEGG: Kyoto encyclopedia of genes and genomes. Nucleic Acids Res 28: 27-30, 2000.

15. Franceschini A, Szklarczyk D, Frankild S, Kuhn M, Simonovic M, Roth A, Lin J, Minguez P, Bork P, von Mering C and Jensen LJ: STRING v9. 1: Protein-protein interaction networks, with increased coverage and integration. Nucleic Acids Res 41 (Detabase Issue): D808-D815, 2013.

16. Saito R, Smoot ME, Ono K, Ruscheinski J, Wang PL, Lotia S, Pico AR, Bader GD and Ideker T: A travel guide to Cytoscape plugins. Nat Methods 9: 1069-1076, 2012.

17. Adamcsek B, Palla G, Farkas IJ, Derényi I and Vicsek T: CFinder: Locating cliques and overlapping modules in biological networks. Bioinformatics 22: 1021-1023, 2006.

18. Derényi I, Palla G, and Vicsek T: Clique percolation in random networks. Phys Rev Lett 94: 160202, 2005.

19. Apweiler R, Attwood TK, Bairoch A, Bateman A, Birney E, Biswas M, Bucher P, Cerutti L, Corpet F, Croning MD, et al: The InterPro database, an integrated documentation resource for protein families, domains and functional sites. Nucleic Acids Res 29: 37-40, 2001.

20. Tew SR, Kwan AP, Hann A, Thomson BM and Archer CW: The reactions of articular cartilage to experimental wounding: Role of apoptosis. Arthritis Rheum 43: 215-225, 2000.

21. Costouros JG and Kim HT: Preventing chondrocyte programmed cell death caused by iatrogenic injury. Knee 14: 107-111, 2007.

22. Khan IM, Palmer EA and Archer CW: Fibroblast growth factor-2 induced chondrocyte cluster formation in experimentally wounded articular cartilage is blocked by soluble Jagged-1. Osteoarthritis Cartilage 18: 208-219,2010.

23. Moore EE, Bendele AM, Thompson DL, Littau A, Waggie KS Reardon B and Ellsworth JL: Fibroblast growth factor-18 stimulates chondrogenesis and cartilage repair in a rat model of injury-induced osteoarthritis. Osteoarthritis Cartilage 13: 623-631, 2005

24. Zhu C, Zhao J, Bibikova M, Leverson JD, Bossy-Wetzel E, Fan JB, Abraham RT and Jiang W: Functional analysis of human microtubule-based motor proteins, the kinesins and dyneins, in mitosis/cytokinesis using RNA interference. Mol Biol Cell 16: 3187-3199, 2005.

25. Min BJ, Kim N, Chung T, Kim OH, Nishimura G, Chung CY, Song HR, Kim HW, Lee HR, Kim J, et al: Whole-exome sequencing identifies mutations of KIF22 in spondyloepimetaphyseal dysplasia with joint laxity, leptodactylic type. Am J Hum Genet 89: 760-766, 2011.
26. Boyden ED, Campos-Xavier AB, Kalamajski S, Cameron TL, Suarez P, Tanackovic G, Andria G, Ballhausen D, Briggs MD, Hartley C, et al: Recurrent dominant mutations affecting two adjacent residues in the motor domain of the monomeric kinesin KIF22 result in skeletal dysplasia and joint laxity. Am J Hum Genet 89: 767-772, 2011.

27. Sun Y, Mauerhan DR, Franklin AM, Norton J, Hanley EN Jr and Gruber HE: Phosphocitrate is potentially a disease-modifying drug for noncrystal-associated osteoarthritis. Biomed Res Int 2013: 326267, 2013.

28. Sun Y, Roberts A, Mauerhan DR, Sun AR, Norton HJ and Hanley EN Jr: Biological activities of phosphocitrate: A potential meniscal protective agent. Biomed Res Int 2013: 726581, 2013.

29. Taylor SS and Mckeon F: Kinetochore localization of murine Bub1 is required for normal mitotic timing and checkpoint response to spindle damage. Cell 89: 727-735, 1997.

30. Davenport JW, Fernandes ER, Harris LD, Neale GA and Goorha R: The mouse mitotic checkpoint gene bublb, a novel bub1 family member, is expressed in a cell cycle-dependent manner. Genomics 55: 113-117, 1999.

31. Sartor H, Ehlert F, Grzeschik KH, Müller R and Adolph S: Assignment of two human cell cycle genes, CDC25C and CCNB1, to $5 \mathrm{q} 31$ and 5q12, respectively. Genomics 13: 911-912, 1992.

32. Zhang C, Wang L, Wu D, Chen H, Chen Z, Thomas-Ahner JM, Zynger DL, Eeckhoute J, Yu J, Luo J, et al: Definition of a FoxA1 Cistrome that is crucial for G1 to S-phase cell-cycle transit in castration-resistant prostate cancer. Cancer Res 71: 6738-6748, 2011.

33. Van Der Kraan PM, Buma P, Van Kuppevelt $T$ and Van Den Berg WB: Interaction of chondrocytes, extracellular matrix and growth factors: Relevance for articular cartilage tissue engineering. Osteoarthritis Cartilage 10: 631-637, 2002.

34. Pfander D, Cramer T, Deuerling D, Weseloh G and Swoboda B: Expression of thrombospondin-1 and its receptor CD36 in human osteoarthritic cartilage. Ann Rheum Dis 59: 448-454, 2000.

35. Cecil DL, Appleton CT, Polewski MD, Mort JS, Schmidt AM, Bendele A, Beier F and Terkeltaub R: The pattern recognition receptor CD36 is a chondrocyte hypertrophy marker associated with suppression of catabolic responses and promotion of repair responses to inflammatory stimuli. J Immunol 182: 5024-5031, 2009.

36. Zheng Q, Zhou G, Morello R, Chen Y, Garcia-Rojas X and Lee B: Type $\mathrm{X}$ collagen gene regulation by Runx 2 contributes directly to its hypertrophic chondrocyte-specific expression in vivo. J Cell Biol 162: 833-842, 2003.

37. Lin AC, Seeto BL, Bartoszko JM, Khoury MA, Whetstone H, Ho L, Hsu C, Ali SA and Alman BA: Modulating hedgehog signaling can attenuate the severity of osteoarthritis. Nat Med 15: 1421-1425, 2009.

38. Komori T: Regulation of bone development and extracellular matrix protein genes by RUNX2. Cell Tissue Res 339: 189-195, 2010.

39. Jin SY, Hong SJ, Yang HI, Park SD, Yoo MC, Lee HJ, Hong MS, Park HJ, Yoon SH, Kim BS, et al: Estrogen receptor-alpha gene haplotype is associated with primary knee osteoarthritis in Korean population. Arthritis Res Ther 6: R415-R421, 2004.

40. Lamas JR, Rodríguez-RodríguezL, Vigo AG, Alvarez-Lafuente R, López-Romero P, Marco F, Camafeita E, Dopazo A, Callejas S, Villafuertes E, et al: Large-scale gene expression in bone marrow mesenchymal stem cells: A putative role for COL10A1 in osteoarthritis. Ann Rheum Dis 69: 1880-1885, 2010. 